La revue La revue pour l'histoire du CNRS

POUR LHISTOIRE DU CNRS $\quad 16 \mid 2007$

L'expertise scientifique

\title{
L'expertise de la sûreté nucléaire en France
}

Un point de vue institutionnel et technique

Cyrille Foasso

\section{(2) OpenEdition}

Journals

Édition électronique

URL : https://journals.openedition.org/histoire-cnrs/1549

DOI : 10.4000/histoire-cnrs.1549

ISSN : 1955-2408

Éditeur

CNRS Éditions

Édition imprimée

Date de publication : 3 avril 2007

ISBN : 978-2-271-06453-0

ISSN : $1298-9800$

Référence électronique

Cyrille Foasso, "L'expertise de la sûreté nucléaire en France », La revue pour l'histoire du CNRS [En ligne], 16 | 2007, mis en ligne le 26 mars 2009, consulté le 20 mai 2021. URL : http://

journals.openedition.org/histoire-cnrs/1549; DOI : https://doi.org/10.4000/histoire-cnrs.1549

Ce document a été généré automatiquement le 20 mai 2021

Comité pour l'histoire du CNRS 


\section{L'expertise de la sûreté nucléaire en France}

Un point de vue institutionnel et technique

Cyrille Foasso

La sécurité en marchant : les pionniers de l'atome et la sécurité

1 La sécurité a, depuis longtemps, été au cœur des préoccupations des ingénieurs et scientifiques qui ont développé l'énergie nucléaire, ce qui n'empêchera pas des remises en cause profondes. Les brevets Joliot avant-guerre décrivent ainsi les principes de fonctionnement d'un réacteur nucléaire et s'interrogent déjà sur les moyens de prévention des accidents.

2 L'expertise nucléaire, ses méthodes, ses concepts, ses structures, sont l'œuvre en France du Commissariat à l'énergie atomique. Créé en octobre 1945, le CEA est l'acteur exclusif des recherches et du développement des applications nucléaires pendant plus de dix ans. Au cours de cette phase, un nombre réduit de spécialistes a la charge à la fois du développement et de la sécurité de leurs projets.

Ce fonctionnement va être remis en cause au milieu des années cinquante. La mise en place de normes et règlements en matière de sécurité par les organismes internationaux (AIEA, ONU, Euratom) ${ }^{2}$, mais aussi les premiers accidents comme celui de Windscale en Grande-Bretagne font prendre conscience de la nécessité absolue de formaliser les questions de sécurité au CEA, tant dans les concepts que dans les structures ${ }^{3}$.

La professionnalisation de l'expert

4 Fin 1957, le responsable scientifique du CEA, le haut-commissaire Francis Perrin, initie une réflexion sur l'organisation de la sûreté nucléaire. Alimentée par les exemples américain, britannique et canadien, elle aboutit à la création, en janvier 1960, d'une Commission de sûreté des installations atomiques (CSIA), chargée d'examiner la sûreté des installations en cours et à venir du Commissariat. Toute construction, mise en fonctionnement ou modification des conditions de fonctionnement d'installations doit désormais être soumise à l'approbation de la CSIA. 
5 La CSIA se réunira tous les trois mois sous la présidence du haut-commissaire entre 1960 et $1970{ }^{4}$. C'est une sorte de «tribunal » au sein duquel « siègent » les différents départements parties prenantes du CEA ${ }^{5}$. La sûreté nucléaire est présentée comme relevant de l'intérêt général de la communauté nucléaire. L'absence d'accident nucléaire est considérée comme une condition sine qua non par le milieu des ingénieurs pour ancrer l'acceptabilité de cette technique dans sa phase de développement. Un premier groupe d'experts à plein temps voit le jour, le Groupe technique de sûreté des piles, qui compte d'abord trois ingénieurs du CEA ${ }^{6}$. Nouvelle profession et nouvelle discipline des techniques nucléaires : la sûreté. Des études spéciales sont lancées pour lever les incertitudes sur certaines questions, voire orienter les projets.

La distinction des rôles est amorcée, sans être totale. Ceci est conforme à la conception CEA de l'expertise : l'expert en sûreté ne doit pas être un gendarme ou un législateur lointain, qui entraverait le développement par l'application de règles trop formelles. Il doit au contraire apporter une aide, collaborer de manière constante et confiante avec les projeteurs. D'ailleurs, le chef du département des études de piles, le département qui conçoit tous les projets de réacteurs au CEA, sera au cours des années soixante en même temps le chef du groupe de sûreté des piles.

Des pratiques prohibées, constitution d'un corpus de méthodes

7 Avec cette spécialisation des experts et la formalisation du processus d'expertise, certaines pratiques tolérées jusque-là sont désormais prohibées: les sécurités manuelles sont remplacées par des sécurités automatiques, des détecteurs de fuites plus efficaces sont installés, les enceintes et leur étanchéité sont renforcées, des filtres sont rajoutés, les responsabilités entre constructeurs, opérateurs sont mieux définies... Ces modifications ne vont pas sans difficultés car elles nécessitent également des changements d'habitude face aux modes de pensée tenaces des scientifiques et techniciens, celui notamment consistant à considérer que, parce qu'ils sont les plus compétents en tant que développeurs, ils doivent d'évidence conserver la haute main sur la sécurité.

8 Une innovation majeure voit également le jour : à l'instar des pratiques américaines, il est désormais fait obligation aux concepteurs de rédiger un « rapport de sûreté » pour obtenir l'autorisation de construction puis de mise en fonctionnement des installations. Ce rapport doit passer en revue toutes les mesures mises en œuvre dans les projets pour parer à tous les scenarii d'accidents envisageables, même les pires. Les arguments et les démonstrations développés par les projeteurs sont alors analysés par les experts de sûreté.

9 L'expertise, ou l'analyse de sûreté est une activité fondamentalement technique. Un accident pouvant provenir d'un défaut de maitrise de la réaction en chaîne ou d'un défaut de refroidissement du réacteur, divers paramètres et leur influence au cours des scenarii accidentels doivent être examinés : coefficients de température du combustible et du modérateur, excédents de réactivité, durées de vie des neutrons, section de capture du fluide de refroidissement, capacité calorifique et conductibilité thermique du modérateur, tenue des gaines à la température et à l'irradiation, processus de rupture des enceintes, capacités de rétention des produits radioactifs par les structures...

10 Un consensus s'établit à l'échelle internationale autour d'une méthode dite déterministe de prévention et d'analyse des accidents, baptisée "défense en profondeur » ou méthode « des barrières » en France, qui examine la tenue de barrières 
successives entre les poisons radioactifs contenus dans le cœur du réacteur et l'environnement dans diverses situations accidentelles. Mais d'autres questions préoccupent les experts de sûreté : considérant que le risque nul n'existe pas, jusqu'où est-il raisonnable de prendre en compte les accidents ? Après un consensus autour de la définition de l'accident maximum crédible, ces réflexions débouchent à la fin des années soixante sur l'approche dite probabiliste du risque...

L'administration des Mines et l'expertise institutionnalisée

11 L'arrivée d'EDF dans le domaine nucléaire va progressivement modifier le schéma de l'expertise et du contrôle de la sûreté nucléaire, sans en bouleverser les principes. En l'absence d'une loi ou d'une réglementation nucléaire en France, aucun texte n'explicitant les modalités d'expertise de la sûreté 7 , la Commission de sûreté du CEA est, de fait, amenée à examiner la sûreté des installations proposées par les industriels. Ainsi, au début des années soixante les ingénieurs chargés des premiers projets nucléaires d'EDF (les trois premières centrales graphite-gaz de Chinon) ont soumis la sûreté de leurs projets à l'examen de la commission de sûreté du CEA, seule instance reconnue pour sa compétence. Mais les rivalités entre les deux organismes pour l'industrialisation du programme nucléaire français à la fin des années soixante vont conduire à une remise en cause de l'hégémonie du CEA dans le processus d'expertise.

12 En juin 1967, un groupe d'experts ad hoc pour la sûreté des réacteurs est instauré ${ }^{8}$ par le ministre de l'Industrie pour l'examen de la centrale de Saint-Laurent-des-Eaux. C'est un groupe tripartite où sont représentés le CEA, EDF et l'industrie. Ce groupe d'experts devient Groupe permanent (GP) en $1972{ }^{9}$. La composition de ce groupe est un savant dosage entre les différents intérêts en présence, mais également entre les différents grands corps d'ingénieurs au sein même du ministère de l'Industrie. Le Groupe permanent est chargé de donner des avis et de faire des propositions au ministre sur les prescriptions techniques générales pour éviter les dangers ou les inconvénients pouvant résulter de la création ou du fonctionnement des installations nucléaires. Le CEA est chargé de mener les études et il est rapporteur des dossiers devant le Groupe permanent. À partir de cette date, le GP réacteurs ${ }^{10}$ sera présidé par les chefs successifs de la sûreté nucléaire au CEA.

13 La formalisation du processus d'expertise se précise puisque l'année suivante, en mars $1973{ }^{11}$, un échelon administratif est institué au sein du ministère de l'Industrie pour l'examen de la sûreté des installations nucléaires. C'est une victoire d'EDF et des industriels qui obtiennent ainsi que le CEA, seul organisme compétent en matière d'expertise nucléaire, ne soit pas juge et partie dans l'analyse des projets de ses concurrents. Le Service central de sûreté des installations nucléaires (SCSIN) démarre avec un effectif réduit de trois ingénieurs des Mines. Le service est responsable de la politique du contrôle de la sûreté nucléaire en France, et il est en particulier chargé d'élaborer une réglementation technique.

14 Si l'autorité en matière de sûreté est désormais aux mains du Service central, le rôle d'expertise technique du CEA est conforté. En effet, le Service central n'est pas doté de moyens propres d'expertise. Il doit faire appel au CEA où un Département de sûreté nucléaire a été créé en 1970 sous la direction de Jean Bourgeois. Le DSN regroupe les compétences du CEA en matière de sûreté: experts en neutronique, thermo hydraulique, mécanique, combustible... soit 300 personnes environ en 1970.

15 Il est chargé d'analyser les dossiers des industriels, de mener les études et les recherches de sûreté qui permettent d'étayer les recommandations pour le service 
central. Le schéma de contrôle de la sûreté nucléaire en France est en place en 1976, avec la création d'un institut d'expertise nucléaire qui regroupe à la fois les experts de sûreté et de radioprotection du CEA : l'Institut de protection et de sûreté nucléaire (IPSN), qui reste administrativement au sein du CEA. L'IPSN comptera jusqu'à 1500 personnes. La stratégie du CEA et de son administrateur général André Giraud a payé. Le scenario américain qui a vu en 1975, au nom de la séparation contrôleur/contrôlé, le démantèlement de l'Atomic Energy Commission en deux entités - l'une chargée du développement (le Department of Energy) et l'autre du contrôle (la Nuclear Regulatory Commission) -, n'aura pas lieu en France. L'argument qui a prévalu est la nécessaire compétence technique des experts, qui ne peut se maintenir qu'au contact des avancées les plus récentes des sciences et techniques nucléaires, c'est-à-dire au sein du CEA.

16 À la fin des années soixante-dix, le schéma du contrôle de la sûreté nucléaire en France est en place: le SCSIN ${ }^{12}$ exerce le pouvoir de décision au nom du ministre de l'Industrie ; il s'appuie sur l'expertise de son "appui technique ", l'IPSN ; il peut en outre convoquer les Groupes permanents d'experts pour certains problèmes techniques de sûreté plus lourds comme la création, la mise en service, le fonctionnement ou la mise à l'arrêt des installations, ou certaines questions qui peuvent constituer une doctrine, comme la gestion des accidents graves.

17 Fort d'un corpus de doctrines, d'un vivier d'experts parmi les plus compétents et d'une assise organisationnelle, il sera bien difficile aux opposants au programme électronucléaire d'EDF de contester la pertinence des décisions de l'administration en matière de sûreté, d'autant que les rapports de ses experts - parfois fort critiques à l'encontre d'EDF - ne sont pas rendus publics.

Encadré

18 Le rôle du CNRS dans l'histoire de l'expertise nucléaire apparaît à travers la réaction suscitée en 1974 parmi les chercheurs en physique nucléaire et corpusculaire (la commission $06 \mathrm{du}$ CNRS) par le rapport Klapisch (IN2P3 Orsay) et Ripka (CEA). Ce rapport prospectif, dans sa dernière partie consacrée à l'énergie nucléaire*, approuvait le vaste programme électronucléaire lancé en 1974 par le gouvernement, ce qui choqua puisque le CNRS n'avait jamais pris de position officielle sur cette question.

La Commission 06 mit sur pied un groupe de travail sur l'énergie nucléaire à l'automne 1974. Un rapport de ce groupe, adopté à l'unanimité par la commission, souligna un «nombre important de problèmes qui restent à résoudre, et notamment du point de vue de la recherche fondamentale ${ }^{* *}$, comme la pollution thermique et radioactive, la sûreté, les déchets. Cette revue des problèmes conduisait le groupe à conseiller « la plus grande prudence » dans le développement de l'industrie nucléaire.

20 Ce sera le début de l'implication d'un certain nombre de scientifiques dans le débat nucléaire, dont certains constitueront le GSIEN (Groupement de scientifiques pour l'information sur l'énergie nucléaire). Alors que la contre-expertise dans le domaine nucléaire s'est surtout développée dans les aspects liés à la radioprotection, ce sera l'une des rares et persistantes voix critiques abordant également les questions de sûreté nucléaire.

21 * DGRST, «Rapport de prospective - Physique corpusculaire, vol.1, Physique nucléaire ", Numéro spécial du Progrès scientifique, 1975, pp. 135-140.

22 ** Groupe de travail de la commission 06, «Le Point. L'énergie nucléaire », Courrier du CNRS, $N^{\circ} 19$, janvier 1976, pp. 25-29, cit. p. 25. 


\section{NOTES}

1.Les autres risques liés à la production d'énergie nucléaire (exposition à la radioactivité en fonctionnement normal, rejets d'effluents, échauffement des rivières, prolifération, déchets...) mériteraient sans doute chacun un développement équivalent. Notons que la prise de conscience du risque lié à la radioactivité et la réglementation de l'exposition remontent bien avant la découverte de la fission de l'atome et la compréhension des mécanismes de production d'énergie d'origine nucléaire.

2.On craint côté CEA que, sous prétexte de sécurité, les agences internationales s'immiscent dans les projets français et promeuvent telle ou telle technologie nucléaire concurrente. Pour défendre leurs intérêts au sein de ces institutions, les personnels du CEA doivent disposer de leurs propres experts sur ces questions.

3.Les Français qui ne s'étaient pas interrogés en propre sur les questions de risque audelà des mesures de précaution, découvrent les conceptions américaines et britanniques en matière de risque majeur nucléaire lors des conférences des Nations Unies de Genève en 1955 et 1958.

4.Cinq sous-commissions seront successivement créées pour préparer les dossiers de la Commission dans les domaines identifiés du risque nucléaire : sûreté des piles, sûreté des masses critiques, sûreté des risques de contamination chimique ou radioactive, sites, transports. L'essentiel des travaux de la CSIA concernera les travaux de la souscommission de sûreté des piles.

5.Sont membres de la CSIA les directeurs du département de production de plutonium, de la direction des applications militaires, de la direction industrielle, de la direction des matériaux et combustibles nucléaires, de la direction de la physique et des piles atomiques, et du service de contrôle des radiations et de génie radioactif.

6.On assiste donc à la fusion entre la personne " experte » et «l'expert » au sens où Philippe Roqueplo entendait distinguer la personne reconnue pour sa compétence de celle mobilisée afin d'établir une expertise. Cf. Philippe Roqueplo, Entre savoir et décision, l'expertise scientifique, Paris, Éditions Inra, 1997.

7.Un décret du 11 décembre 1963 (décret n63-1228) issu de la discussion sur la loi du 2 août 1961 relative à la lutte contre les pollutions atmosphériques et les odeurs instituera un régime spécifique pour la création des installations nucléaires dites de base (INB) : elles doivent recevoir une autorisation (et non plus seulement être déclarées) du ministère de l'Industrie après avis d'une Commission interministérielle des installations nucléaires de base et accord du ministère de la Santé. Mais le décret reste muet sur ce qui constitue le fond de l'autorisation, c'est-à-dire la procédure d'examen de la sûreté. Seul expert en France, la CSIA poursuit l'examen de la sûreté des installations des industriels.

8.Ses membres sont nommés conjointement par le ministre de l'Industrie et le ministre délégué, chargé de la Recherche scientifique et des questions atomiques et spatiales. 9.Décision relative à la création et au fonctionnement de groupes permanents chargés d'étudier les aspects techniques et la sûreté des installations nucléaires de base, signée du ministre du Développement industriel et scientifique le 15 mars 1972.

10.Trois Groupes permanents ont en fait été créés auprès du ministre du Développement industriel et scientifique : l'un pour les réacteurs, un second pour les accélérateurs de particules et un dernier pour toutes les autres installations. 
11.Décret n73-278 du 13 mars 1973 portant sur la création d'un Conseil supérieur de la sûreté nucléaire et d'un Service central de sûreté des installations nucléaires au ministère du Développement industriel et scientifique.

12.De petit service au départ, l'échelon administratif deviendra Direction de la sûreté des installations nucléaires (DSIN) en 1988, puis Direction générale de la sûreté nucléaire et de la radioprotection (DGSNR) en 2002, comptant une centaine d'ingénieurs au niveau central et autant en régions, avec triple tutelle des ministères de l'Industrie, de l'Écologie et de la Santé.

\section{RÉSUMÉS}

Le potentiel catastrophique de dissémination de quantités considérables de produits radioactifs en cas d'accident de réacteur nucléaire allait faire naître, à la fin des années 1950, un corpus de méthodes et une famille d'experts dévolus à la prévention de ce type de risques ${ }^{1}$, désignée à partir de 1960 sous le vocable de «sûreté nucléaire ". Retour sur la genèse de l'expertise de la sûreté nucléaire en France.

At the end of the 50's, the potential risk of dissemination of a huge quantity of radioactivity in case of a nuclear accident leads to formalize the expertise of nuclear risk prevention. Back to the genesis of the nuclear power safety expertise in France.

\section{AUTEUR \\ CYRILLE FOASSO}

Cyrille Foasso est ingénieur diplômé de l'INSA de Lyon, docteur en histoire moderne et contemporaine. Chargé de collection au Musée des arts et métiers (CNAM), il est responsable de la collection des instruments scientifiques contemporains. 